\title{
Interferência da luz: uma correlação entre o experimento de Young e a litografia interferométrica
}

Light interference: a correlation between Young's experiment and interference lithography

\author{
J. W. Menezes ${ }^{*} \sqrt{0}$, C. Valsecchi ${ }^{10}$ \\ ${ }^{1}$ Universidade Federal do Pampa, Campus Alegrete, RS, Brasil.
}

Recebido em 18 de maio de 2020. Revisado em 11 de junho de 2020. Aceito em 18 de junho de 2020.

\begin{abstract}
Este trabalho tem como objetivo relacionar o experimento de Young com uma técnica experimental de fabricação de estruturas periódicas conhecida como litografia interferométrica de dois feixes. A relação entre os experimentos foi feita em termos de distribuição de intensidade, considerando uma abordagem vetorial, onde foi utilizado como ponto de partida a superposição de duas ondas planas. Nos limites considerados no experimento de Young, os resultados mostram que a distribuição de intensidade de ambos os experimentos coincidem. Além disso, este estudo possibilita ampliar a discussão da interferência luminosa, particularmente o uso deste fenômeno em aplicações tecnológicas.
\end{abstract}

Palavras-chave: óptica, interferência, Experimento de Young, litografia interferométrica.

\begin{abstract}
This work aims to relate Young's experiment with an experimental technique for periodic structures fabrication known as two-beam interference lithography. The relationship between the experiments was made in terms of intensity distribution, considering a vectorial approach, where the superposition of two plane waves was used as a starting point. Within the limits considered in Young's experiment, the results show that the intensity distribution of both experiments coincide. In addition, this study provides a broader discussion on light interference, particularly the use of this phenomenon in technological applications.
\end{abstract}

Keywords: optics, interference, Young's experiment, interference lithography.

\section{Introdução}

A abordagem do experimento de Young (EY) nos livros didáticos tradicionais de física e componentes curriculares normalmente é introduzida tendo como principal objetivo a demonstração da natureza ondulatória da luz [1][2]. Além disso, a descrição matemática do experimento mostra que é possível determinar com boa precisão o comprimento de onda de uma fonte laser. Nesta abordagem, duas fontes pontuais $S_{0}$ e $S_{1}$ estão separadas por uma distância d e um anteparo está localizado a uma distância $\mathrm{D}$ das fontes, conforme mostra a figura 1. A superposição das duas frentes de onda provenientes de $\mathrm{S}_{0}$ e $\mathrm{S}_{1}$ cria um padrão de máximos e mínimos de intensidade luminosa no espaço (ou num anteparo), também chamado de padrão de interferência. Neste modelo, considera-se d muito pequeno e D muito grande (comparado com d), consequentemente, em um ponto $\mathrm{P}$, a superposição dos feixes provenientes das fontes $\mathrm{S}_{0}$ e $\mathrm{S}_{1}$ podem ser consideradas paralelas [1][2]. A distribuiçao de intensidade $(I)$ do padrão de interferência, considerando-se $d \ll D$ de foma que $\operatorname{sen} \theta=\operatorname{tg} \theta=\frac{x}{D}$ é dada pela seguinte equação:

$$
I=2 I_{0}\left(1+\cos \left(\frac{2 \pi d}{\lambda_{0} D} x\right)\right)
$$

*Endereço de correspondência: jacsonmenezes@unipampa.edu.br

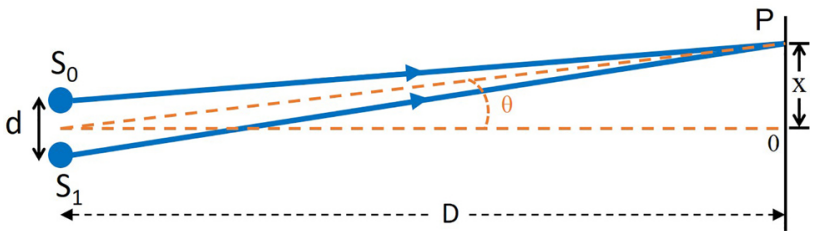

Figura 1: Esquema mostrando o experimento de dupla fenda de Young. Os feixes $S_{0}$ e $S_{1}$, provenientes de uma única fonte externa, se superpõem num anteparo e um padrão de máximos e mínimos de luz se forma ao longo do eixo $x$.

onde $I_{0}$ corresponde a intensidade de luz de cada fonte $\mathrm{S}_{0}$ e $\mathrm{S}_{1}$ e $\lambda_{0}$ corresponde ao comprimento de onda da luz monocromática incidente. Para os parâmetros considerados, a periodicidade $\left(\Lambda=\frac{d x}{d m}\right.$, distância entre dois máximos ou dois mínimos de ordem $m$ consecutivos) é dada pela seguinte equação:

$$
\Lambda=\frac{\lambda_{0} D}{d}
$$

Assim, utilizando a equação 2, a equação 1 pode ser reescrita como:

$$
I=2 I_{0}\left(1+\cos \left(\frac{2 \pi x}{\Lambda}\right)\right)
$$


A técnica de litografia interferométrica (LI) é uma ferramenta que permite gerar padrões periódicos em grandes áreas e com custo baixo quando comparada com outras técnicas de fabricação, tais como feixe de elétrons e feixe de íons focalizados [3][4]. Padrões periódicos 2D podem ser fabricados para aplicações em dispositivos, como por exemplo cristais fotônicos e estruturas plasmônicas [5] [6]. A LI de dois feixes utiliza essencialmente a mesma ideia do experimento de Young, como pode ser visto na Figura 2. A técnica, amplamente descrita em [7-9], consiste na superposição de duas ondas planas provenientes de um mesmo laser, gerando um padrão de interferência no espaço. Se um material fotossensível (anteparo) é colocado na região de superposição dos feixes, o padrão de luz é transferido para este material. Após um processo de revelação do material fotossensível, o padrão de luz é convertido em um padrão em relevo [7-9].

Para a litografia interferométrica, as simplificações em relação aos parâmetros d e D do experimento de Young não são válidas. Isso ocorre uma vez que d pode ter dimensões comparáveis a $\mathrm{D}$, de forma que os feixes interferentes em um ponto $\mathrm{P}$ qualquer não sejam paralelos, ou seja, o problema algébrico da soma dos campos elétricos do experimento de Young se torna um problema vetorial na LI.

Neste sentido, este trabalho tem como objetivo relacionar matematicamente a distribuição de intensidade de luz no EY com a LI de dois feixes e mostrar que nos limites dados pelo EY, as equações de distribuição de intensidade luminosa dos dois experimentos se tornam equivalentes. Além disso, este estudo visa ampliar a discussão do fenômeno de interferência considerando sua utilização em aplicações tecnológicas, indo além dos textos encontrados nos livros tradicionais de física.

\section{Interferência óptica}

\subsection{Superposição de duas ondas planas}

A teoria da interferência óptica é baseada no princípio da superposição de campos eletromagnéticos [10][11]. De acordo com este princípio, o campo elétrico $\vec{E}$ em um ponto do espaço, devido a superposição de vários campos

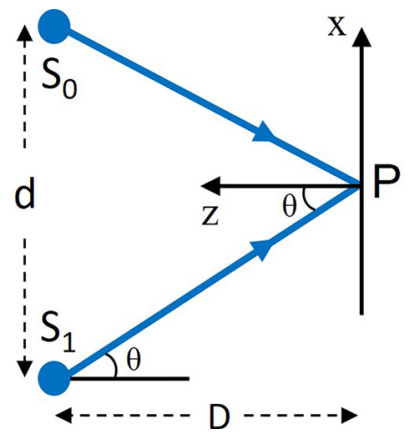

Figura 2: Esquema mostrando o experimento de litografia interferométrica.
$\left(\overrightarrow{E_{1}}, \overrightarrow{E_{2}} \overrightarrow{E_{3}}, \ldots\right)$ produzidos por diferentes fontes, é dada pela soma vetorial destes campos, ou seja:

$$
\vec{E}=\overrightarrow{E_{1}}+\overrightarrow{E_{2}}+\overrightarrow{E_{3}}+\ldots
$$

O princípio é uma consequência do fato de que as equações de Maxwell no vácuo são equações diferenciais lineares [10][11].

O padrão de interferência resultante da superposição de duas ondas planas $\left(\vec{E}_{1}\right.$ e $\left.\vec{E}_{2}\right)$ pode ser representado pela expressão [11]:

$$
I=\frac{c}{8 \pi}\left|\overrightarrow{E_{1}}+\overrightarrow{E_{2}}\right|^{2}=\frac{c}{8 \pi}\left(\overrightarrow{E_{1}}+\overrightarrow{E_{2}}\right) \cdot\left(\overrightarrow{E_{1}^{*}}+\overrightarrow{E_{2}^{*}}\right)
$$

onde os campos $\vec{E}_{1}$ e $\overrightarrow{E_{2}}$, de mesma polarização, são dados pelas equações:

$$
\begin{gathered}
\overrightarrow{E_{1}}=\overrightarrow{E_{0}} \exp \left(\overrightarrow{k_{1}} \vec{r}-\omega t\right) \\
\overrightarrow{E_{2}}=\overrightarrow{E_{0}} \exp \left(\overrightarrow{k_{2}} \cdot \vec{r}-\omega t\right)
\end{gathered}
$$

com $\overrightarrow{E_{0}}$ sendo o vetor amplitude do campo elétrico de frequência angular $\omega, \vec{r}$ é o vetor posição $(\vec{r}=x \hat{x}+y \hat{y}+z \hat{z})$ e, $\overrightarrow{k_{1}}$ e $\overrightarrow{k_{2}}$ são os vetores de onda, cujo módulo é dado por:

$$
\left|\overrightarrow{k_{i}}\right|=k_{0}=\frac{2 \pi}{\lambda_{0}}
$$

onde $\lambda_{0}$ é o comprimento de onda da fonte de luz (laser) utilizado.

Substituindo as equações 6 e 7 em 5, a distribuição de intensidade de luz pode ser simplificada por:

$$
I=2 I_{0}\left(1+\cos \left(\vec{k}_{1} \vec{r}-\vec{k}_{2} \vec{r}\right)\right.
$$

Desta forma, a geometria, a direção e a periodicidade do padrão de interferência dependem dos vetores $\overrightarrow{k_{1}}$ e $\overrightarrow{k_{2}}$.

\subsection{O caso de estruturas periódicas $2 \mathrm{D}$ : simulação e fabricação}

Muitas aplicações tecnológicas, tais como dispositivos de cristais fotônicos e materiais plasmônicos, necessitam de um padrão bidimensional (2D) de estruturas dielétricas ou metálicas gravadas em um substrato [5][6]. Um padrão $2 \mathrm{D}$ é aquele que as estruturas tem periodicidade em duas dimensões (plano xy por exemplo). A Figura 3 mostra um esquema que permite gerar tais padrões bidimensionais utilizando a superposição de duas ondas planas.

Neste esquema, o plano formado pelos feixes interferentes é perpendicular ao plano da amostra (anteparo) onde pode ser gravado este padrão. Os vetores de onda incidentes $\overrightarrow{k_{1}}$ e $\overrightarrow{k_{2}}$ formam o mesmo ângulo $\theta$ em relação à bissetriz (B), ou seja, são simétricos em relação ao eixo z. Este ângulo $\theta$ define o período $\Lambda$ das franjas do padrão de interferência. Nas simulações, para gerar um padrão $2 \mathrm{D}$, faz-se necessário duas exposições ao padrão de interferência, girando um padrão em relação ao outro de um ângulo $\beta$ qualquer, diferente de zero. De acordo 


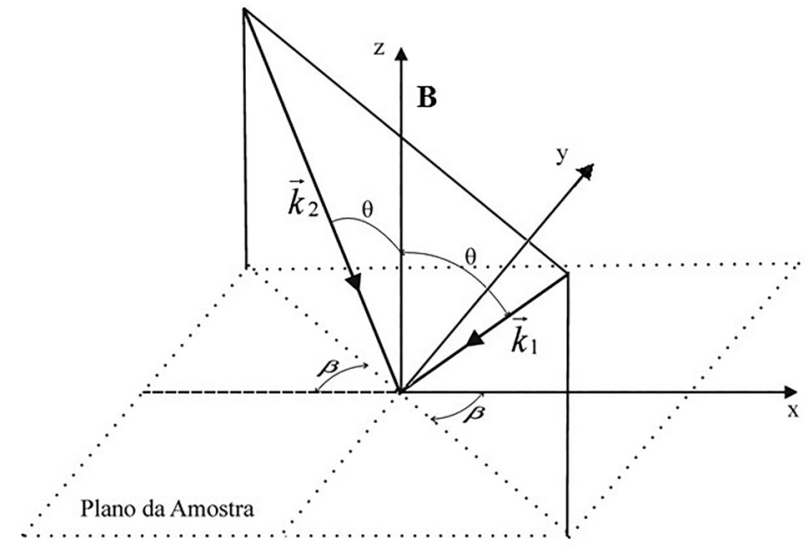

Figura 3: Esquema dos feixes interferentes utilizado para descrever um padrão bidimensional. A geometria resultante depende apenas da escolha de $\beta$ entre duas exposições.

com o sistema de coordenadas mostrado na Figura 3, os vetores de onda $\overrightarrow{k_{1}}$ e $\overrightarrow{k_{2}}$ podem ser escritos como:

$$
\begin{aligned}
\overrightarrow{k_{1}} & =k_{0} \operatorname{sen} \theta \cos \beta \hat{x}-k_{0} \operatorname{sen} \theta \operatorname{sen} \beta \hat{y}-k_{0} \cos \theta \hat{z} \\
\overrightarrow{k_{2}} & =-k_{0} \operatorname{sen} \theta \cos \beta \hat{x}+k_{0} \operatorname{sen} \theta \operatorname{sen} \beta \hat{y}-k_{0} \cos \theta \hat{z}
\end{aligned}
$$

Considerando a equação 8 e substituindo as equações 10 e 11 na equação 9 , tem-se:

$$
I=2 I_{0}\left[1+\cos \left(\frac{4 \pi}{\lambda_{0}} \operatorname{sen} \theta(\cos \beta x-\operatorname{sen} \beta y)\right)\right]
$$

Como dito anteriormente, o ângulo $\theta$ define a periodicidade do padrão de interferência. De acordo com a equação 12 , considerando por simplicidade $\beta=0$, a periodicidade é dada pela seguinte equação:

$$
\Lambda=\frac{\lambda_{0}}{2 \operatorname{sen} \theta}
$$

Assim, utilizando a equação 13, a equação 12 pode ser reescrita como:

$$
I=2 I_{0}\left[1+\cos \left(\frac{2 \pi}{\Lambda}(\cos \beta x-\operatorname{sen} \beta y)\right)\right]
$$

Esta equação permite gerar diversos tipos de padrões $2 \mathrm{D}$ de acordo com o número de exposições e ângulos de rotação $\beta$ entre as exposições. No caso de múltiplas exposições (ME), a intensidade final será dada pela somatória das intensidades para cada ângulo $\beta$ [7]. Levando isto em consideração, a equação 14 pode ser escrita como:

$$
I_{M E}=\sum_{i} I_{\beta i}
$$

onde $\mathrm{i}=1,2,3, \ldots, n$ corresponde a uma, duas, três, $n$ exposições para os correspondentes ângulos $\beta$. Em particular, se a amostra for girada em torno do eixo z de $90^{\circ}$ entre duas exposições, ou seja, na primeira exposição, $\beta_{1}=0^{\circ}$ e na segunda exposição $\beta_{2}=90^{\circ}$, uma rede $2 \mathrm{D}$ de simetria quadrada será formada. Se a amostra for girada de $60^{\circ}$ entre as exposições $\left(\beta_{1}=30^{\circ}, \beta_{2}=90^{\circ}\right)$, uma rede $2 \mathrm{D}$ com simetria hexagonal será gerada. A Figura 4 mostra os resultados das simulações, utilizado o software Mathematica [12] com script de autoria própria, dos padrões de luz resultantes (curvas de iso-dose, equivalente a tempos diferentes de exposição) para estes dois casos. Considerando $\lambda_{0}=458 \mathrm{~nm}$ e $\theta=19,1^{0}$ ( $\mathrm{D}=1,0 \mathrm{~m}$ e $\mathrm{d} \sim 0,7 \mathrm{~m}$ ) em ambas as exposições, de acordo com a equação 13 isto fornece uma periodicidade do padrão de interferência de $\Lambda=700 \mathrm{~nm}$. Para estes mesmos parâmetros, de acordo com as equações do EY, a periodicidade seria de $\sim 660 \mathrm{~nm}$. Esta diferença entre os dois experimentos aumenta se a periodicidade das franjas diminui. Isso ocorre porque quando a periodicidade diminui, o tratamento escalar deixa de ser válido. A título de exemplo, mantendo d fixo, se a distância D for reduzida para $\mathrm{D}=0,2 \mathrm{~m}$, a periodicidade por LI será o dobro da periodicidade dada no EY. Como pode ser observado ainda na Figura 4, a periodicidade do padrão de interferência $(\Lambda)$ pode ser diferente da periodicidade final $(a)$ da rede gravada. Esta periodicidade $(a)$ depende da geometria final devido a combinação das duas exposições. Por exemplo, para a geometria quadrada, a peridicidade $a$ coincide com a periodicidade $\Lambda$, enquanto que para uma geometria hexagonal, a superposição de dois padrões com periodicidade $\Lambda=700 \mathrm{~nm}$ fornece uma periodicidade da rede de $a=808 \mathrm{~nm}\left(a=\frac{2}{\sqrt{3}} \Lambda\right)$.

Para verificar os resultados da simulação, foram gravadas estruturas $2 \mathrm{D}$ - utilizando o mesmo período e os mesmos ângulos de rotação das simulações mostradas na Figura 4 - em fotorresinas positivas SC 1827 depositadas sobre vidro. A preparação das amostras, exposições e caracterização foram feitas utilizando os métodos descritos na referência [8]. A Figura 5 mostra imagens dos padrões 2D gravados, vistos de cima, por microscopia eletrônica de varredura. Comparando-se as estruturas obtidas experimentalmente com os padrões simulados, observa-se um excelente acordo, o que comprova o modelo aqui descrito.

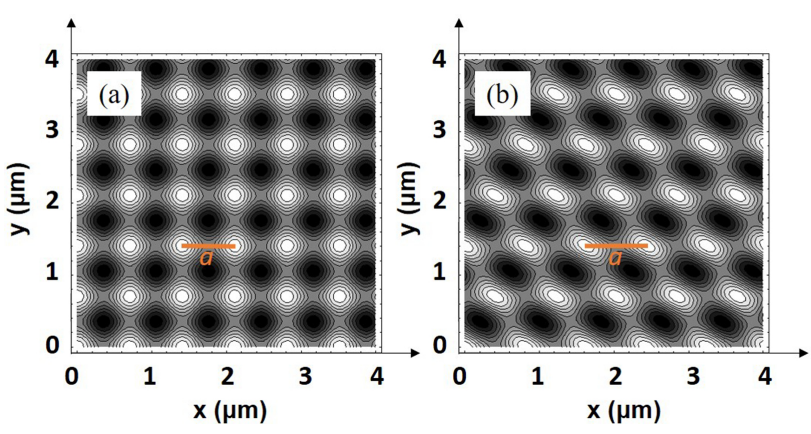

Figura 4: Simulação do padrão de luz resultante da superposição de dois padrões de interferência idênticos com período das franjas de $\Lambda=0,7 \mu \mathrm{m}$. (a) $\beta_{1}=0^{\circ}$ e $\beta_{2}=90^{\circ}$, (b) $\beta_{1}=30^{\circ}$ e $\beta_{2}=90^{\circ}$ 

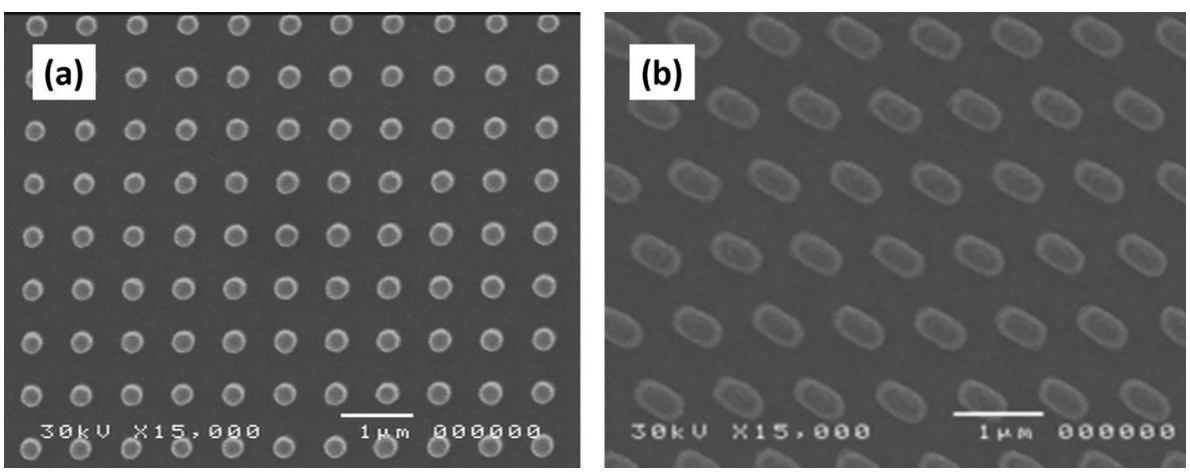

Figura 5: Padrões 2D gravados na fotorresina positiva SC 1827 utilizando a superposição de dois padrões de interferência: (a) $\beta_{1}=0^{\circ}$ e $\beta_{2}=90^{\circ}$ e, (b) $\beta_{1}=30^{\circ}$ e $\beta_{2}=90^{\circ}$

\section{Equivalência do modelo de LI com o EY}

Considerando $\beta=0$ (ver Figura 3), os vetores de onda $\overrightarrow{k_{1}}$ e $\overrightarrow{k_{2}}$ podem ser escritos como:

$$
\begin{aligned}
\overrightarrow{k_{1}} & =k_{0} \operatorname{sen} \theta \hat{x}-k_{0} \cos \theta \hat{z} \\
\overrightarrow{k_{2}} & =-k_{0} \operatorname{sen} \theta \hat{x}-k_{0} \cos \theta \hat{z}
\end{aligned}
$$

Substituindo as equações acima em 9 , juntamente com 8 e 13, tem-se:

$$
I=2 I_{0}\left[1+\cos \left(\frac{2 \pi}{\Lambda} x\right)\right]
$$

Como pode ser observado, as equações 18 e 3 são equivalentes. Porém, para que as distribuições de intensidade dos dois experimentos sejam iguais, as equações que envolvem a periodicidade em ambas as situações também devem ser equivalentes. Isso só é possível se for considerado o limite em que $\operatorname{sen} \theta=\operatorname{tg} \theta$, caso em que $d \ll D$. Nesta condição, de acordo com a Figura 2, $\operatorname{tg} \theta=\operatorname{sen} \theta=\frac{d / 2}{D}$. Esta condição aplicada na equação 13 resulta em uma periodicidade $\Lambda=\frac{\lambda_{0} D}{d}$, a qual equivale à periodicidade dada no experimento de Young. Este resultado mostra que a abordagem da interferência luminosa do EY pode ser tratada de forma vetorial, sendo que nos limites em que as fontes se aproximam e o anteparo se afasta, o problema resulta no caso escalar como discutido nos livros didáticos tradicionais de física.

\section{Conclusões}

Os resultados descritos neste trabalho mostram que é possível fazer uma analogia entre o experimento de Young e a técnica de fabricação de dispositivos conhecida como litografia interferométrica. Neste sentido, a formulação matemática deve ser tratada do ponto de vista vetorial. Com a modelagem vetorial foi possível prever padrões geométricos que se aplicam em situações tecnológicas, como na fabricação de estruturas periódicas 2D. Simulações utilizando o modelo proposto foram comparadas com padrões bidimensionais gerados em laboratório mostrando uma ótima concordância. Para efeitos de comparação, aplicando os limites descritos no experimento de Young ao modelo vetorial, ambas as equações se tornam equivalentes. Considerando tal modelo, professores e alunos poderão explorar o EY não somente para descrever a natureza ondulatória da luz, mas também como uma ferramenta que permite modelar padrões de interferência que podem ser aplicados em pesquisas de ponta.

\section{Referências}

[1] D. Halliday, R. Resnick e J. Walker, Física: Óptica e Física Moderna (LTC, Rio de Janeiro, 2006), v. 4.

[2] P. A. Tipler e G. Mosca, Física para cientistas e engenheiros. Eletricidade e magnetismo, óptica (LTC, Rio de Janeiro, 2009), v. 2.

[3] S. Kim, H. Chong, R.M. De La Rue, J.H. Marsh e A.C. Bryce, em Conference on Lasers and ElectroOptics/Quantum Electronics and Laser Science Conference (Maryland, 2003).

[4] D. Freeman, S. Madden e B. Luther-Davies, Opt. Express 13, 3079 (2005).

[5] J.D. Joannopoulos, R.D. Meade e J.N. Winn, Photonic Crystals (Princeton University Press, New Jersey, 1995).

[6] T.W. Ebbesen, H.J. Lezec, H.F. Ghaemy, T. Thio e P.A. Wolff, Nature 391, 667 (1998).

[7] N.D. Lai, W.P. Liang, J.H. Lin, C.C. Hsu e C.H. Lin, Opt. Express 13, 9605 (2005).

[8] C. Valsecchi, L.E.G. Armas e J.W. Menezes, Sensors 19, 2182 (2019).

[9] C. Lu e R.H. Lipson, Laser Photonics Rev. 4, 568 (2010).

[10] G.R. Fowles, Introduction to modern optics (Dover, New York, 1989).

[11] E. Hecht, Optics (Addison-Wesley, Boston, 2001), $4^{\text {th }}$ ed. 